\title{
ON ITERATIVE FEEDBACK TUNING AND DISTURBANCE REJECTION USING SIMPLE NOISE MODELS
}

\author{
Bo Wahlberg* \\ *S3-Automatic Control, KTH, SE 10044 Stockholm, \\ Sweden. Email: bo.wahlberg@s3.kth.se
}

\begin{abstract}
The objective of this contribution is to discuss three basic control design methods for disturbance rejection. The key idea is to use a simple fixed continuous time noise model as a design variable to specify the desirable closed loop properties, e.g., the closed loop bandwidth. We will study three closely related controller design methods based on the noise model, namely minimum variance control, weighted minimum variance control and a loop shaping design procedure proposed by Skogestad and Postlethwaite. All these methods lead to simple controller structures, where the transfer function of the input/output relation is the only unknown part.

The main motivation of this study has been tuning of controllers for disturbance rejection by means of iterative feedback tuning (IFT). Here, the controller parameters are found by minimizing a specified cost function using gradient search techniques. The gradients are estimated using data from iterative experiments. This is a rather difficult problem if no external input excitation is allowed. Import factors for good results are the controller parametrization and the choice of cost function.

We propose an IFT cost function based on a simple output signal weighting tailored to the fixed noise model controller structure. This leads to robust procedure for iterative feedback tuning of a controller for disturbance rejection. The approach is illustrated on a simple example. Copyright (C) 2005 IFAC
\end{abstract}

Keywords: control tuning; iterative feedback tuning; disturbance rejection control oriented identification; identification; minimum-variance regulator.

\section{INTRODUCTION}

This paper is motivated by practical experience using Iterative Feedback Tuning (IFT) for designing controllers for disturbance rejection. The control performance is measured by a specified

\footnotetext{
1 Research was partially supported by the Swedish Research Council.
}

cost function, and the objective is to find the controller that within a given structure minimizes this cost. This is done by gradient search techniques, where the gradients are estimated using data from multiple experiments. See (Hjalmarsson, 2002) for a thorough overview. IFT works well if it is possible to use external excitation signals to obtain reliable gradient estimates. It is, however, more difficult to use IFT if the only excitation is the 
external disturbances, which should be rejected by the feedback. It is then most important to have a simple controller structure with as few free parameters as possible together with a cost function that reflects the control specifications, c.f. (Lequin et al., 2003).

We will use continuous time models to describe the underlying ideas, even if the feedback and tuning most often are done using sampled data. The reason is that this makes it easier to present the basic concepts.

Consider the feedback control system in Fig. 1.

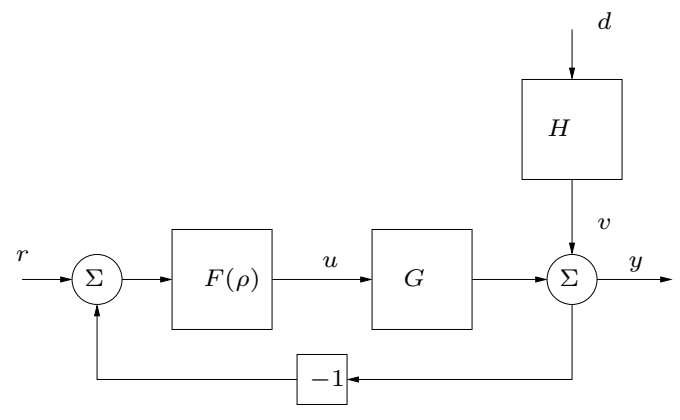

Fig. 1. Feedback control system.

The transfer function of the system is given by $G(s)$. The plant input is denoted by $u(t)$, the output by $y(t)$ and $r(t)$ is the reference signal. The controller is specified by the transfer function $F(s, \rho)$, where $\rho$ is the controller parameters to be determined. The objective of the feedback control is to reject the additive output disturbance $v(t)$. We will assume that the reference signal is zero.

The disturbance $v(t)$ is modelled as the output from a noise filter $H(s)$, excited by the signal $d(t)$. The transfer function from $d(t)$ to $y(t)$ will be called the weighted sensitivity function, and equals

$$
S(s) H(s), \quad S(s)=\frac{1}{1+G(s) F(s)},
$$

where $S(s)$ is the sensitivity function.

We will make extensive use of the following simple noise filter model

$$
H(s)=\frac{s+\omega_{0} S_{0}}{s S_{0}}, \quad \omega_{0}, S_{0}>0 .
$$

The noise filter $H(s)$ behaves as an integrator for low frequencies, the amplitude of the frequency response $|H(i \omega)| \approx 1$ at the frequency $\omega=\omega_{0}$ and the high frequency gain is $1 / S_{0}$. This means that it is most important to have effective feedback in the frequency range $\left[0, \omega_{0}\right]$, but that there also is high frequency noise. This will avoid large controller gains at high frequencies (differentiation). The noise model parameters should be viewed as design variables, where $\omega_{0}$ determines the desired bandwidth. The choice of $S_{0}$ is less important, but should be chosen larger than one.
The control performance will be measured by the discrete time cost function

$$
\begin{aligned}
J(\rho)=\frac{1}{N} \sum_{k=1}^{N} & {\left[\left(W_{T}(q) y(k T, \rho)\right)^{2}\right.} \\
& \left.+\lambda(\Delta(q) u(k T, \rho))^{2}\right],
\end{aligned}
$$

where $T$ is the sampling interval, $W_{T}(q)$ is a discrete time weighting filter and $\Delta(q)=1-q^{-1}$ the difference operator. Here $q$ denotes the shift operator $q y(k T)=y((k+1) T)$. The parameter $\lambda>0$ balances the output regulation with the changes in the input signal. We have stressed that the input and output are functions of the controller parameters $\rho$. The task is to find the controller that minimizes the cost function $J(\rho)$. Iterative Feedback Tuning (IFT) is a method to minimize such a cost function by using information from iterative experiments.

The paper is organized as follows. Section 2 discusses controller design for disturbance rejection. IFT is then introduced in Section 3, while Section 4 describes how to combine the disturbance rejection control design ideas described in Section 2 with IFT to obtain a simple data driven tuning method. This method is illustrated on a numerical example in Section 5. Finally, Section 6 concludes the paper.

\section{CONTROLLER DESIGN FOR DISTURBANCE REJECTION}

Next, we will review three simple model based controller design methods, namely minimum variance control, weighted minimum variance control and a loop shaping design procedure.

Assumption: In order to simplify the presentation we will assume that the transfer function $G(s)$ is of relative degree one, is asymptotically stable and is minimum phase (all zeros strictly in the left half plane).

\subsection{Minimum Variance Control}

Assume that the input $d(t)$ to the noise filter $H(s)$ is continuous time white noise, i.e., has constant power for all frequencies, see e.g. (Åström, 1970). This is mainly a technical assumption to obtained well-posed continuous time stochastic control problems. However, since the variance of continuous time white noise is infinite, one has to be careful when deriving the continuous time minimum variance control law. By assuming $H(\infty) \neq$ 0 (semi-proper) the minimum variance controller equals

$$
F_{M V}(s)=\frac{1}{G(s)}\left[\frac{H(s)}{H(\infty)}-1\right] .
$$


Notice that $[H(s) / H(\infty)-1]$ will be strictly proper (more poles than zeros). The assumption that $G(s)$ has relative degree one will guarantee a proper controller (no direct differentiation of $y(t)$ ). If the relative degree of $G(s)$ is higher than one, we have to include a low-pass filter in the controller to make it proper. Possible unstable poles of $G(s)$ shared by $H(s)$ can also be handled. The closed loop relation will be $y(t)=H(\infty) d(t)$, i.e., the output will also be white noise.

For the simple noise filter (2)

$$
H(s)=\frac{s+\omega_{0} S_{0}}{s S_{0}} \Rightarrow \frac{H(s)}{H(\infty)}-1=\frac{\omega_{0} S_{0}}{s} .
$$

Hence, the loop transfer function $G(s) F_{M V}(s)$ is just an integrator, where the gain determines the bandwidth. Here, the weighted sensitivity (1) equals $S_{M V}(s) H(s)=1 / S_{0}$. The bandwidth of the sensitivity function $S_{M V}(s)$ will be around $S_{0} \omega_{0}$. The input size increases with $S_{0}$, and it is well known that minimum variance controller may have poor robustness if the bandwidth is chosen too high. It should however be noted that minimum variance control using appropriate values of $S_{0}$ gives well behaved controllers. It is even possible to de-tune the controller by choosing $S_{0}<1$.

\subsection{Weighted Minimum Variance Control}

One common way to introduce frequency weighting is to minimize variance of the filtered output, i.e., the variance of $W(s) y(t)$, where $W(s)$ typically is a low pass filter. The optimal controller equals

$$
F_{W M V}(s)=\frac{1}{G(s)}\left[\frac{W(s) H(s)}{W(\infty) H(\infty)}-1\right] .
$$

The corresponding weighted sensitivity is

$$
S_{W M V}(s) H(s)=\frac{W(\infty) H(\infty)}{W(s)} .
$$

The discrete time version of this approach is studied from a control performance supervision perspective in (Horch, 2000), where the use of a sampled version of the first order filter

$$
W(s)=\frac{1+s / b}{1+s / \mu}, \quad b>>\mu,
$$

is proposed. The corresponding discrete time approach is called de-tuned minimum variance control in (Åström and Wittenmark, 1995). It should, however, be noted that frequency weighting gives more high gain control at low frequencies, since the low frequency disturbances are further suppressed while the gain of $S_{W M V}(s) H(s)$ for high frequencies still is $H(\infty)$.

\subsection{A Loop Shaping Design}

Assume that the noise input $d(t)$ is scaled so that $|d(t)|<1$. Let also the output $y(t)$ be scaled in such a way that the control objective is to make $|y(t)|<1$ for all disturbances $|d(t)|<1$. This can be translated to the frequency domain condition

$$
|S(i \omega) H(i \omega)|<1, \quad \forall \omega .
$$

In (Skogestad and Postlethwaite, 1996) the simple approximate solution $G(s) F(s)=H(s)$ is proposed. The frequency domain condition $(5)$ is then satisfied at the frequencies where $|H(i \omega)|>>1$. The controller $F(s)=H(s) / G(s)$ may have to be modified to increase the gain at low frequencies and to obtain acceptable phase and gains margins around the cross-over frequency. See Section 2.6.4 in (Skogestad and Postlethwaite, 1996) for details. The resulting controller will be of the form

$$
F_{L S}(s)=\frac{1}{G(s)} H(s) \frac{s+a}{s} F_{L P}(s),
$$

where $F_{L P}(s)$ is a low-pass filter to make the controller proper. Here it is also assumed that the zeros of $G(s)$ are in the left-half plane. If this is not the case, possible "unstable" zeros have to be mirrored into the left half plane or omitted when forming $1 / G(s)$ in the controller. The same holds for time delays.

For the simple noise model (2) this design gives

$$
S_{L S}(s) H(s)=\frac{s+\omega_{0} S_{0}}{\left(S_{0}+1\right) s+\omega_{0} S_{0}} .
$$

This transfer function has static gain 1 , a pole at $s=-\omega_{0}\left(1+S_{0}\right) / S_{0}$ and a zero at $s=-\omega_{0} S_{0}$. A typical value of $S_{0}$ is 2 , which means that the bandwidth of $S_{L S}(s) H(s)$ will be slightly less than $\omega_{0}$. This should be compared with the minimum variance controller which gives a bandwidth around $S_{0} \omega_{0}$. Also here $\omega_{0}$ is the most important tuning parameter. The purpose of the PI-term is to obtain better disturbance rejection for low frequencies. A reasonable choice is $a=\omega_{0} / 5$. A typical bandwidth of $F_{L P}(s)$ is $10 \omega_{0}$.

\section{ITERATIVE FEEDBACK TUNING}

The gradient of the cost function (3) with respect to the controller parameters is given by

$$
\begin{aligned}
& \frac{d}{d \rho} J(\rho)= \\
& \frac{2}{N} \sum_{k=1}^{N}\left(\left[W_{T}(q) y(k T, \rho)\right]\left[W_{T}(q) \frac{d}{d \rho} y(k T, \rho)\right]\right. \\
& \left.\quad+\lambda[\Delta(q) u(k T, \rho)]\left[\Delta(q) \frac{d}{d \rho} u(k T, \rho)\right]\right) .
\end{aligned}
$$


Consider the controller parameter update

$$
\rho(i+1)=\rho(i)-\gamma_{i} R^{-1}(i) \frac{d}{d \rho} J(\rho(i)),
$$

where $\gamma_{i}$ is the step-length and $R(i)$ is a positive definite matrix. A good choice is the Hessian estimate

$$
\begin{aligned}
& R(\rho)= \\
& \begin{aligned}
\frac{2}{N} \sum_{k=1}^{N} & \left(\left[W_{T}(q) \frac{d}{d \rho} y(k T, \rho)\right]\left[W_{T}(q) \frac{d}{d \rho} y(k T, \rho)\right]^{T}\right. \\
& \left.+\lambda\left[\Delta(q) \frac{d}{d \rho} u(k T, \rho)\right]\left[\Delta(q) \frac{d}{d \rho} u(k T, \rho)\right]^{T}\right),
\end{aligned}
\end{aligned}
$$

which should be modified to $R(i)=R\left(\rho_{i}\right)+\delta I$, $\delta>0$, to handle ill-conditioned cases. The steplength $\gamma_{i}$ is typically around 1 .

The main problem is how to find the gradients $\frac{d}{d \rho} y(t, \rho)$ and $\frac{d}{d \rho} u(t, \rho)$. The key idea of IFT is to estimate these signals using iterative experiments.

Let $u_{1}(t, \rho)$ and $y_{1}(t, \rho)$ be the input and output from the feedback system in Fig. 1 with the controller $F(\rho)$ and with reference signal $r_{1}(t)=0$. Now make a first experiment to measure these signals. Then perform a second experiment with reference signal $r_{2}(t)=-K_{1} y_{1}(t)$ (where $K_{1} \geq 1$ is a gain factor) and denote the corresponding input and output by $u_{2}(t, \rho)$ and $y_{2}(t, \rho)$, respectively. We then have

$$
\begin{aligned}
y_{1}(t) & =\frac{1}{1+G F(\rho)} v_{1}(t) \\
y_{2}(t) & =\frac{G F(\rho)}{1+G F(\rho)}\left[-K_{1} y_{1}(t)\right]+\frac{1}{1+G F(\rho)} v_{2}(t) \\
& =\frac{G F(\rho)}{1+G F(\rho)}\left[\frac{-K_{1}}{1+G F(\rho)} v_{1}(t)\right] \\
& +\frac{1}{1+G F(\rho)} v_{2}(t) \\
& \approx K_{1} \frac{G F(\rho)}{1+G F(\rho)}\left[\frac{-1}{1+G F(\rho)} v_{1}(t)\right] .
\end{aligned}
$$

The last approximation is based on the assumption that the gain $K_{1}$ is large enough so that the $v_{2}(t)$ related noise contribution can be neglected. Now the gradient (with no reference signal) equals

$$
\begin{aligned}
& \frac{d}{d \rho} y_{1}(t, \rho)=-\frac{G \frac{d}{d \rho} F(\rho)}{(1+G F(\rho))^{2}} v_{1}(t) \\
& =\frac{\frac{d}{d \rho} F(\rho)}{F(\rho)} \frac{G F(\rho)}{1+G F(\rho)} \frac{-1}{1+G F(\rho)} v_{1}(t) .
\end{aligned}
$$

Comparing with (6) gives the gradient estimate

$$
\frac{d}{d \rho} y(t, \rho)=\frac{1}{K_{1}} \frac{\frac{d}{d \rho} F(\rho)}{F(\rho)} y_{2}(t, \rho) .
$$

The gradient of the input signal can be derived in a similar way. For a more detailed derivation taking the stochastic properties of the noise into account see (Hjalmarsson, 2002).

Our experience is that it is quite difficult to tune controllers using IFT for disturbance rejection. The disturbance is the only excitation signal and the objective of the controller is reject this signal. In the recent work, (Hildebrand et al., 2004), on IFT for disturbance rejection, the gain $K_{1}$ used in the feedback experiment is optimized so as to minimize the variance of the gradient estimate error, subject to power constraints on the signals. Typically, the cost function (3) is quite insensitive to the choice of regulator. This is of course good from a control perspective, but difficult when tuning the controller.

\section{DESIGN ISSUES}

When using IFT to tune a controller for disturbance rejection it is important:

- To have a simple controller structure with as few free parameters as possible.

- To have a cost function that gives an optimal controller which satisfies the specification without using too large input signals.

Here we have proposed the use the simple noise model

$$
H(s)=\frac{s+\omega_{0} S_{0}}{s S_{0}}
$$

with the two design parameters $\omega_{0}$ and $S_{0}$. This leads to a controller structure of the form

$$
F_{L S}(s)=\frac{1}{G(s)} H(s) \frac{s+a}{s} F_{L P}(s),
$$

or

$$
F_{W M V}(s)=\frac{1}{G(s)}\left[\frac{W(s) H(s)}{[W(\infty) H(\infty)}-1\right] F_{L P}(s),
$$

of which the minimum variance control is a special case $\left(W=1, F_{L P}=1\right)$. Here we also have to specify the low-pass filter $F_{L P}(s)$ and the parameters $a$ or $\mu$ (which are closely related). The main unknown part in the controller is the transfer function $G$. Hence, it is natural to use the model parameters of $G$ as controller parameters, c.f. indirect adaptive control (Åström and Wittenmark, 1995). From a control perspective it is often enough to use very simple models, as first or second order transfer functions or even just a gain.

To connect the disturbance rejection control design with IFT an obvious candidate for cost function is

$$
\begin{aligned}
J(\rho)=\frac{1}{N} \sum_{k=1}^{N} & {\left[\left(W_{T}(q) y(k T, \rho)\right)^{2}\right.} \\
& \left.+\lambda(\Delta(q) u(k T, \rho))^{2}\right],
\end{aligned}
$$


with $W_{T}$ a first order low pass filter with bandwidth around $\omega_{0}$. The parameter $\lambda$ is more difficult to choose, but reflects the balance between control performance and input size. Weighted minimum variance control corresponds to $\lambda=0$.

\section{EXAMPLE}

Here we will study the properties of the proposed approach for a very simple example, namely

$$
G(s)=\frac{b}{s+1}, b=1, \quad H(s)=\frac{s+2}{2 s} .
$$

We will assume that only the gain $b$ of the transfer function is unknown. This is, of course, an oversimplification, but viewing the model as only a controller design variable we have fixed everything in the controller except the gain.

The specifications mean that we need active control up to around $\omega_{0}=1 \mathrm{rad} / \mathrm{s}$. Introduce $K=$ $1 / b$, which will correspond to the controller gain. We will study the three controllers:

$$
\begin{aligned}
F_{L S}(s) & =\frac{1}{G(s)} H(s) F_{L P}(s) \\
& =K(s+1) \frac{s+2}{2 s} \frac{10}{s+10}, \\
F_{M V}(s) & =\frac{1}{G(s)}\left[\frac{H(s)}{H(\infty)}-1\right]=\frac{2 K(s+1)}{s}, \\
F_{W M V}(s) & =\frac{1}{G(s)}\left[\frac{W(s) H(s)}{W(\infty) H(\infty)}-1\right] \\
& =\frac{K(11 s+20)}{s}, \\
W(s) & =\frac{1+s / 10}{(1+s)}
\end{aligned}
$$

and the variance and weighted variance costs

$$
\begin{aligned}
\bar{J}_{M V}(K) & =\mathrm{E}\left\{(y(k T, K))^{2}\right\}, \\
\bar{J}_{W M V}(K) & =\mathrm{E}\left\{\left(W_{T}(q) y(k T, K)\right)^{2}\right\},
\end{aligned}
$$

where $W_{T}$ is the sampled version of $W(s)$. Notice that the gain of the weighted minimum variance controller for low frequencies is a factor of ten larger than for the two other cases. This is to further suppress low frequency noise and results in a larger input signal.

In order to illustrate the properties of the three controller structures and the two cost functions, we calculate $\bar{J}_{M V}(K)$ and $\bar{J}_{W M V}(K)$ as functions of $K$, with the sampling interval $T=0.01$ and with $\{d(t)\}$ being white noise with variance 1 . The continuous time transfer functions are approximated by Euler forward.

In Fig. 2 the variance is plotted as function of the controller gain $K$ for the three controllers. The weighted variance cost is given in Fig. 3, and Fig. 4 contains a zoomed version of $\bar{J}_{W M V}(K)$ for the controller structure $F_{W M V}$.

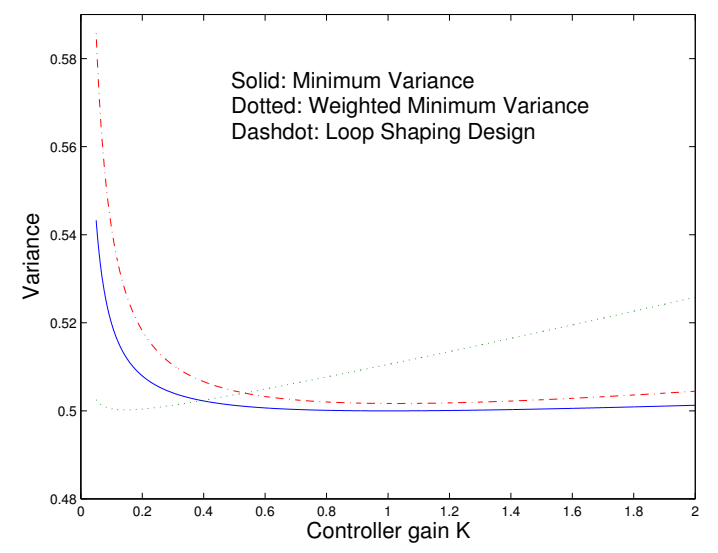

Fig. 2. The variance $\bar{J}_{M V}(K)$ for the three controller structures.

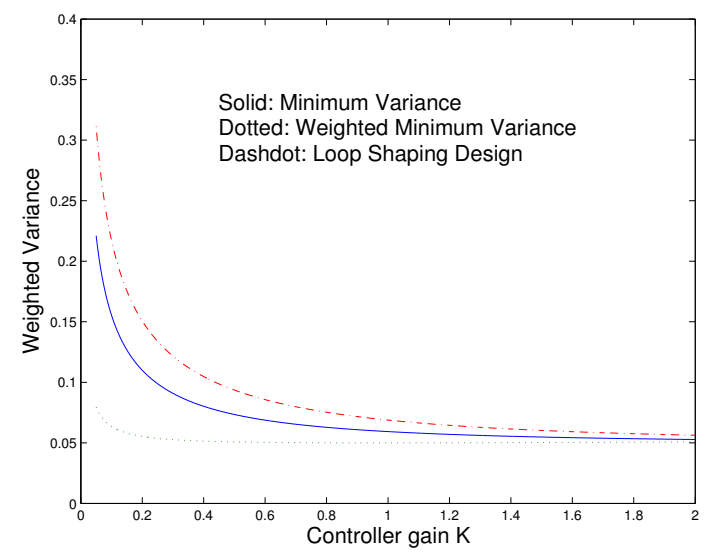

Fig. 3. The weighted variance $\bar{J}_{W M V}(K)$ for the three controller structures.

In order to evaluate the corresponding input power, the variance of the delta input signal

$$
\bar{J}_{u}(K)=\mathrm{E}\left\{(\Delta(q) u(k T, K))^{2}\right\}
$$

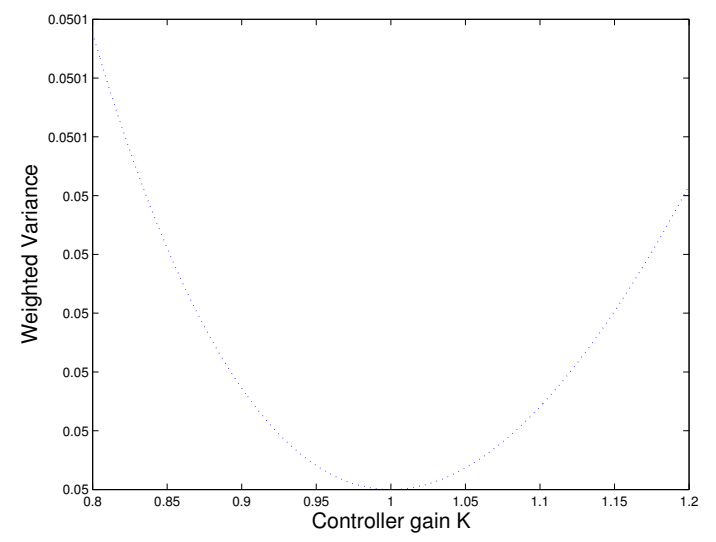

Fig. 4. Zoomed version of weighted variance $\bar{J}_{W M V}(K)$ for $F_{W M V}(s)$. Notice the scale of the $\mathrm{y}$-axis. 


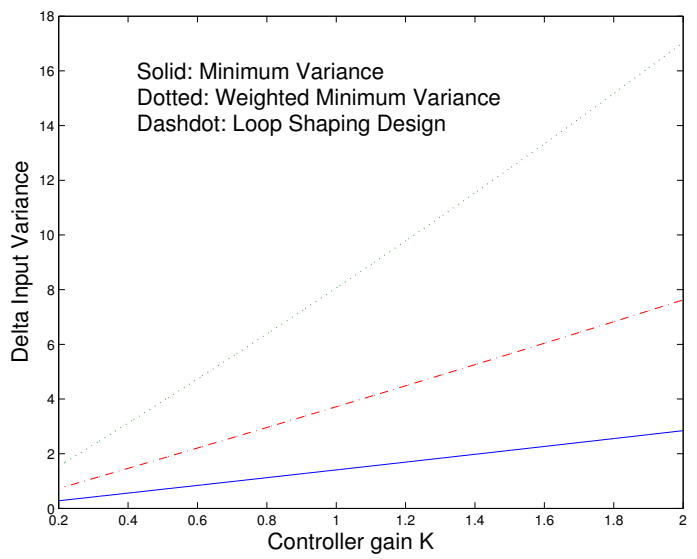

Fig. 5. The delta input signal variance $\bar{J}_{u}(K)$ for the three controller structures.

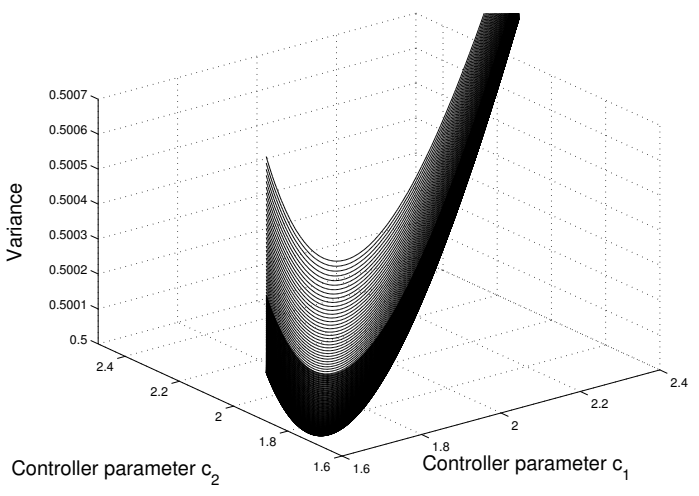

Fig. 6. The variance $\bar{J}_{m v}(K)$ for the controller structure $F_{C}=\left(c_{1} s+c_{2}\right) / s$.

is plotted for the three different controllers as a function of $K$ in Fig. 5. The input energy is lowest for the minimum variance controller and highest for the weighted minimum variance controller as explained in Section 2.

The overall observation is that all cost functions are quite flat around the minimum and hence rather difficult to minimize using gradient search. The good news are that they all are quite robust to the choice of $K$. Also notice that all three controller structures give comparable results.

Next, the variance cost function for the unconstrained controller parametrization is plotted in Fig. 6. It is rather difficult see the details of this 3-dimensional plot. However, the norm of second derivative (Hessian) of the cost function is a measure the flatness around the minimum, and is also directly related the performance of corresponding numerical optimization techniques. Here the norm of the inverse Hessian at the minimum equals 2500. Also notice that variance is quite insensitive to variations in the controller parameters.

\section{CONCLUSIONS}

The objective of this contribution has been to study simple controller structures for disturbance rejection. The work is inspired by industrial applications of tuning controller for disturbance rejection using IFT. If the controller should be tuned on-line using adaptive control or iterative feedback tuning, it is most important to have as few free parameters as possible. It is also important to be able to predict the performance of the control system, e.g., for condition monitoring of control loops.

We have discussed three very simple methods to design a controller for disturbance rejection. The designs are based on two user's choices, namely $\omega_{0}$ and $S_{0}$, of which $\omega_{0}$ is the most important one. The model $G(s)$ of the input output transfer function is needed to determine the controller. One possibility is to estimate the transfer function using system identification methods, see e.g. (Ljung, 1987). An alternative that we have studied is to use iterative feedback tuning (IFT).

\section{Acknowledgement}

The author would like to thank Håkan Hjalmarsson for valuable comments on this work and Peter Uddenfelt for industrial collaboration.

\section{REFERENCES}

Åström, K. J. (1970). Introduction to stochastic control theory. Academic Press.

Åström, K. J. and B. Wittenmark (1995). Adaptive Control. Addison and Wesley.

Hildebrand, R., A. Lecchini, G. Solari and M. Gevers (2004). Prefiltering in iterative feedback tuning: optimization of the prefilter for accuracy. IEEE Transactions on Automatic Control 49, 1801 - 1805.

Hjalmarsson, H. (2002). Iterative feedback tuning -an overview. International Journal on Adaptive Control and Signal Processing 16, 373395.

Horch, A. (2000). Condition Monitoring of Control Loops. PhD thesis. Department of Signals, Sensors and System, KTH.

Lequin, O., M. Gevers, M. Mossberg, E. Bosmans and L. Triest (2003). Iterative feedback tuning of pid parameters: comparison with classical tuning rules. Control Engineering Practice 11, $1023-1033$.

Ljung, L. (1987). System Identification: Theory for the User. Prentice-Hall. Englewood Cliffs, N.J.

Skogestad, S. and I. Postlethwaite (1996). Multivariable Feedback Control. Johns Wiley \& Sons. 